\title{
Separation of Water-in-Oil Emulsions by Electrostatic Field at the Elevated Temperature
}

\author{
Hak Seung Lee ${ }^{1^{*}}$, Chaolun Zheng ${ }^{1}$ and Bao Yang
}

Department of Mechanical Engineering, College Park University of Maryland, USA

"Corresponding author: Hak Seung Lee and Bao Yang Department of Mechanical Engineering, College Park University of Maryland, USA, Tel: 301-405-6007, E-mail: kobegator@gmail.com; baoyang@umd.edu

Rec Date: July 24, 2018; Acc Date: August 13, 2018; Pub Date: August 20, 2018

Copyright: (2018 Lee HS, et al. This is an open-access article distributed under the terms of the Creative Commons Attribution License, which permits unrestricted use, distribution, and reproduction in any medium, provided the original author and source are credited.

\begin{abstract}
Separation of water from oil emulsions has been a significant subject in the petroleum industry and chemical processing. In this paper, we report experimental studies on separation of water from water-in-sunflower oil emulsions under a combined treatment of a radial electric field and elevated temperature. The effects of voltage and temperature on separation time are investigated experimentally and theoretically. It is found that the increase in voltage and/or temperature can significantly reduce the separation time and the residual water in the emulsion. For example, the application of the $1 \mathrm{kV}$ voltages can reduce the separation time by $5-10$ times and decrease the residual water concentration by 4-5 times when compared to the process without electric fields. Increasing the process temperature to $90^{\circ} \mathrm{C}$ can also reduce the separation time by $5-10$ times and decrease the residual water concentration by 5-6 times when compared to the processes at $25^{\circ} \mathrm{C}$. The modeling analysis suggests that the enhanced separation performance can be mainly attributed to the reduced viscosity at elevated temperature and the accelerated droplet coalescence under the radial electric field. An optimized condition is also found to balance the energy consumption and separation efficiency.
\end{abstract}

Keywords: Water/Oil; Emulsions; Electrode; Petroleum and chemical industries

\section{Introduction}

The efficient removal of water from oils has been an important issue in petroleum and chemical industry for many decades [1,2]. There are many different aqueous coalescence techniques which are targeted to remove water from the water-in-oil emulsion: gravity, centrifugal, chemical treatment, heating, electrostatic, mechanical, filtration, and so on [2-4].

Electrostatic coalescence was invented for the petroleum-related industries, and this method uses electric fields to promote the coalescence of small water droplets in water-in-oil emulsions2. In electric fields, small water droplets move towards each other or collide with each other due to the electrostatic forces, such as dipole-dipole interaction and dielectrophoretic force, acting on them. As a result, the merging and coalescence of those droplets will increase the droplet size and eventually lead to settling and separation of water from the oil phase.

The electrostatic separation can be further expedited by using elevated temperature because some properties such as density, viscosity, and dielectric constant become more favorable for separation as the temperature increases. Especially, the viscosity of the oil decreases rapidly at the high temperature, which accelerates the movement and the coalescence of the water droplets $[2,5]$.

In this paper, a separation system is built by using a homemade cylindrical cone-shaped separator equipped with coaxial cylindrical electrodes. The electrostatic field in combination with heat treatment is utilized to accelerate the separation of water from the oil emulsion. The effects of electric voltage, frequency, and temperature on the separation time and concentration of residual water in the emulsion are investigated experimentally. The influences of separation time on residual water concentration and the power consumption of different separation methods are also investigated in this paper.

Sunflower oil is used as a replacement of medium crude oil due to its similar properties [6]. Crude oil contains many natural surfactants such as asphaltenes, resins and fatty acids $[7,8]$. Sodium dodecyl sulfate (SDS) is selected in this paper as the surfactant for sunflower oil emulsions [9]. Water-in-sunflower oil emulsions with wt. $\left(\mathrm{H}_{2} \mathrm{O}\right)=5 \%$ and $10 \%$ are investigated in this paper. The residual water concentrations (\%) in oil phase are measured to compare the qualities of separated oils.

\section{Theoretical calculations}

The droplet movements in electric fields with various temperatures are investigated theoretically [10-15]. The balances of the forces acting on a pair of droplets are indicated in Figure 1. Dipole-dipole and gravitational forces are mainly considered in this analysis. The velocities and collision times of the droplets are theoretically estimated with different temperatures, droplet sizes, electric strengths, and distances between two droplets. As shown in Figure 1, horizontal electric force $\left(\mathrm{F}_{\mathrm{e}} \mathrm{l}\right)$ is created because electrodes are located vertically in our system, while the gravity $\left(F_{g}\right)$ acts vertically to the ground. The flow speeds of the droplets, which are divided into horizontal direction $\mathrm{V}_{\mathrm{H}}$ and vertical direction $\mathrm{V}_{\mathrm{V}}$, can generate the forces of flow resistance $\left(F_{V}\right)$. The forces of flow resistance are also divided into horizontal $\left(\mathrm{F}_{\mathrm{V}, \mathrm{H}}\right)$ and vertical $\left(\mathrm{F}_{\mathrm{V}, \mathrm{V}}\right)$ directions. 


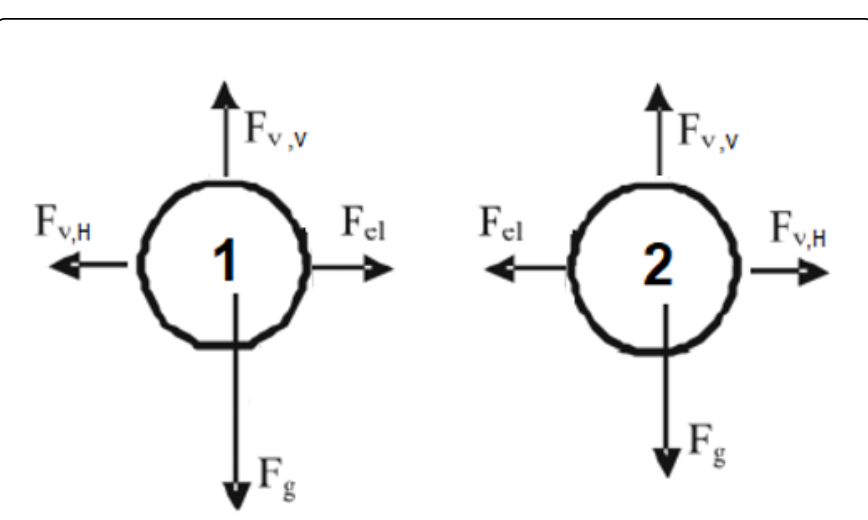

Figure 1: The balance of the forces acting on a pair of droplets in horizontal electric fields.

The force of gravity $\left(\mathrm{F}_{\mathrm{g}}\right)$ on the water droplets with radius a and water density $\rho w$, dispersed into a continuous oil phase of density $\rho$ is expressed in Equation 1. The forces of flow resistance, which are the Stokes forces, through the continuous viscosity phase $\mu$ o along with the velocities $\mathrm{V}_{\mathrm{H}}$ and $\mathrm{V}_{\mathrm{V}}$ are indicated in Equations 2 and 3.

$$
\begin{array}{rlr}
F_{g} & =\frac{4}{3} \pi a^{3}\left(\rho_{w}-\rho_{0}\right) g & (1) F_{v, v}=6 \pi \mu_{0} a V_{V} \\
F_{V, H} & =6 \pi \mu_{0} A V_{H}(3)
\end{array}
$$

where $\varepsilon$ is a permittivity of the medium surrounding oil, $\mathrm{E}_{\mathrm{o}}$ is the strength of the electric field and 1 is the distance between the droplet centers. The radii a of the two droplets are assumed same in this Equation.

$$
F_{e l}=\frac{24 \pi \varepsilon E_{0}^{2} a^{6}}{l^{4}}=\frac{24 \pi \varepsilon E_{0}^{2} a^{2}}{(l / a)^{4}}(4)
$$

where $\varepsilon$ is a permittivity of the medium surrounding oil, $\mathrm{E}_{\mathrm{o}}$ is the strength of the electric field and 1 is the distance between the droplet centers. The radii a of the two droplets are assumed same in this Equation.

\section{Vertical movement by gravity}

First, the vertical movement of water droplets is investigated. In the vertical movement, the force of gravity $\left(\mathrm{F}_{\mathrm{g}}\right)$ along with the force of vertical flow resistance $\left(\mathrm{F}_{\mathrm{v}, \mathrm{v}}\right)$ is considered. For a droplet, the balance of forces in the vertical position can deliver the Equation 5 by using the Equations 1 and 2. The Equation 7 for $\mathrm{dV}_{\mathrm{V}} / \mathrm{dt}$ is obtained from the Equations 5 and 6.

$$
\begin{gathered}
m \frac{d V_{v}}{d t}=\frac{4}{3} \pi a^{3}\left(\rho_{w}-\rho_{0}\right) g-6 \pi \mu_{0} a V_{v} \\
\text { (6) } \frac{d V_{V}}{d t}=\frac{\left(\rho_{w}-\rho_{0}\right) g}{\rho_{w}}-\frac{4.5 \mu_{0} V_{V}}{a^{2} \rho_{w}} \text { (7) }
\end{gathered}
$$

Considering that this is a pseudo-steady state $\left(\mathrm{dV}_{\mathrm{V}} / \mathrm{dt} \approx 0\right)$, the vertical velocity $\left(\mathrm{V}_{\mathrm{V}}\right)$ can be calculated in Equation 8 . The sedimentation time $\left(t_{V}\right)$ of the droplet is also obtained in the Equation 10 with the vertical distance $h$.

$$
\begin{aligned}
V_{V} & =\frac{a^{2}\left(\rho_{w}-\rho_{0}\right) g}{4.5 \mu_{0}} \quad \text { (8) } \frac{d h}{d t}=V_{V}=\frac{a^{2}\left(\rho_{w}-\rho_{0}\right) g}{4.5 \mu_{0}} \\
(9) t_{v} & =\frac{4.5 \mu_{0} h}{a^{2}\left(\rho_{w}-\rho_{0}\right) g} \\
m \frac{d V_{H}}{d t} & =24 \pi \varepsilon E_{0}^{2} a^{6} l^{-4}-6 \pi \mu_{0} a V_{H}
\end{aligned}
$$

The vertical velocity $\left(\mathrm{V}_{\mathrm{V}}\right)$ of water droplets can be affected by several factors such as the densities of water and oil $\left(\rho_{\mathrm{w}}-\rho_{\mathrm{o}}\right)$, oil viscosity $\left(\mu_{o}\right)$, and the radius (a) of the water droplets as indicated in Equation 8. When considering sunflower oil as a continuous oil phase, the density and viscosity are affected by the temperature as shown in Figure 2. Therefore, it is determined that the precipitation speed of water droplet is mainly affected by the operating temperature and the droplet size, and this speed can be increased by the increment of the temperature as well as the droplet size.
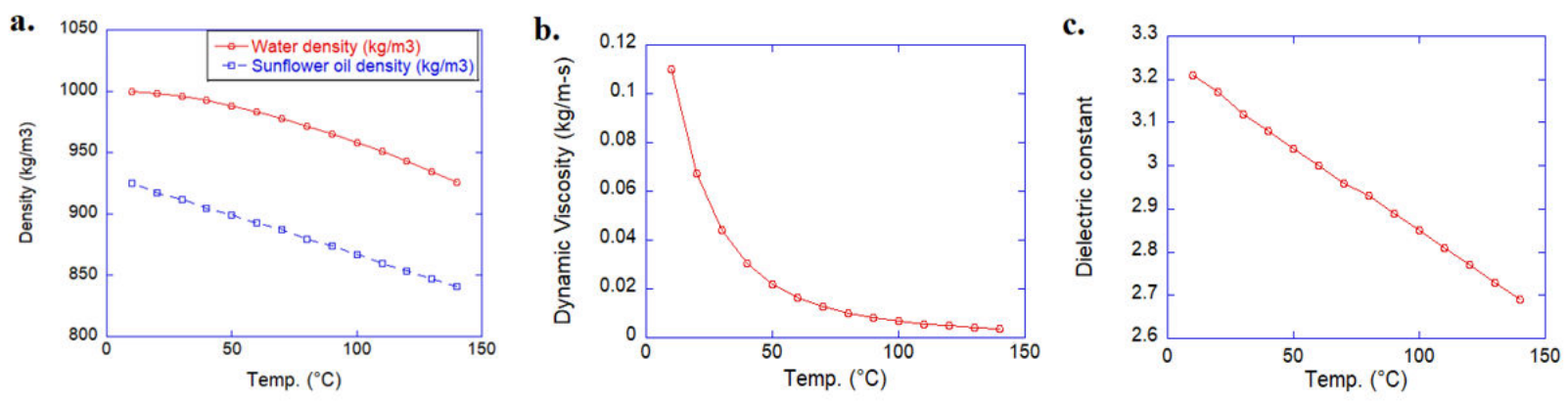

Figure 2: Changes of properties with temperature $\left(10-140^{\circ} \mathrm{C}\right)$ : (a) Densities $(\mathrm{kg} / \mathrm{m} 3)$ of water and sunflower oil, (b) Dynamic viscosities of sunflower oil, (c) Dielectric constants of sunflower oil.

The sedimentation times of the water droplets are analytically calculated with various temperatures and droplet sizes. The vertical distance (h) is assumed to be $10 \mathrm{~cm}$. The precipitation time of the droplets decreases as droplet size and temperature increase, as indicated in Figure 3a. For example, the precipitation time of water droplet is decreased by $5-10$ times with high temperature $\left(90^{\circ} \mathrm{C}\right)$ when compared to the room temperature $\left(25^{\circ} \mathrm{C}\right)$. This reduced precipitation 
time of the droplets can expedite the separation speed of the water-inoil emulsions.

\section{Horizontal movement by electric fields}

Next, the horizontal movement of the water droplets by the electric fields is studied. In the horizontal movement, the electric force of the droplet $\left(\mathrm{F}_{\mathrm{el}}\right)$ along with the force of horizontal flow resistance $\left(\mathrm{F}_{\mathrm{vH}}\right)$ is considered as shown in Figure 1. The balance of the forces on the droplet in the horizontal direction can generate the Equation 11. The Equation 12 of $\mathrm{dV}_{\mathrm{H}} / \mathrm{dt}$ is obtained from the Equations 6 and 11 .

The horizontal velocity $\left(\mathrm{V}_{\mathrm{H}}\right)$ can be also calculated by considering a pseudo-steady state $\left(\mathrm{dv}_{\mathrm{h}} / \mathrm{dt} \approx 0\right)$ assumption as seen in Equation 13 . The collision time $\left(\mathrm{t}_{\mathrm{c}}\right)$ calculated by integrating Equation 14 is expressed in Equation 15. The collision time is influenced by several factors such as $\varepsilon, \mathrm{E}_{\mathrm{o}}, \mu_{\mathrm{o}}$ and $\mathrm{Va}$, among which dielectric constant $(\varepsilon)$ and viscosity $\left(\mu_{\mathrm{o}}\right)$ are deeply influenced by the operating temperature. Therefore, temperature and electric strength can be considered as main factors to determine the collision time with assuming fixed l/a ratio.
The effects of temperature and electric strength on the collision time are numerically calculated. The collision time decreases as temperature and electric strength increase as shown in Figure 3b. For instance, the collision time of the water droplets are reduced by $5-10$ times with high temperature $\left(90^{\circ} \mathrm{C}\right)$ compared to the result at room temperature $\left(25^{\circ} \mathrm{C}\right)$.

From the results of the theoretical calculation, the precipitation and collision times of water droplets in the oil phase is significantly related to the increment of the temperature and electric force. The precipitation and collision times are reduced by 5-10 times by increasing the process temperature to $90^{\circ} \mathrm{C}$ when compared to the process under the same electric field at $25^{\circ} \mathrm{C}$. This reduced coalescence time can accelerate the separation speed of water-in-oil emulsion. The effect of temperature and electric force will be also determined by water/oil separation tests to validate the results of analytical considerations. a.

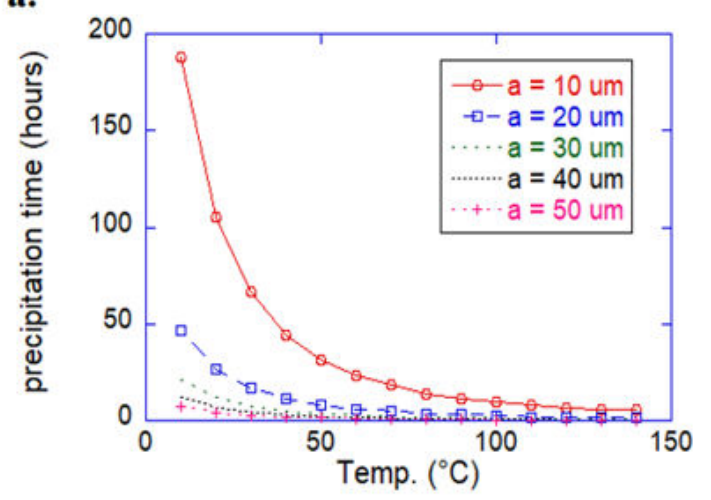

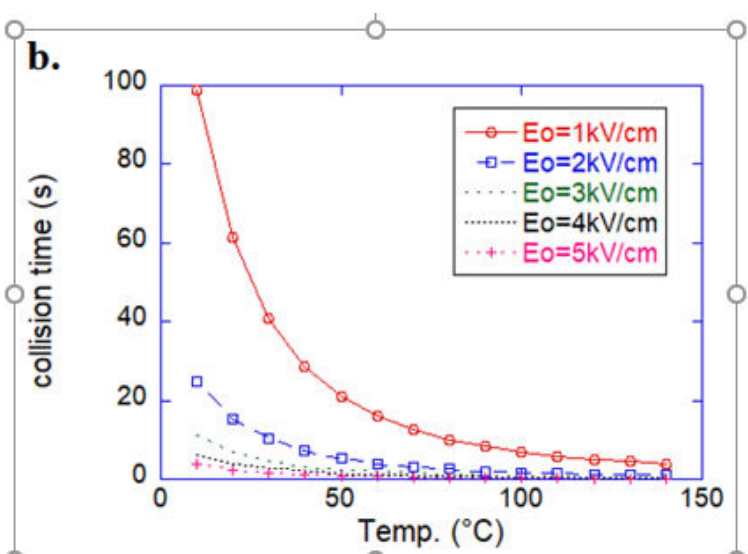

Figure 3: Precipitation and collision time of the water droplets under the influence of temperature $\left(10-140^{\circ} \mathrm{C}\right)$ : (a) Precipitation time (hours) with various droplet sizes, (b) Collision time (sec) with various electric strengths.

\section{Experimental Setup}

\section{Overall setup}

Separation of water-in-sunflower oil emulsions is carried out using an experimental setup as shown in Figure 4. The system consists of a function generator, a voltage amplifier, a temperature-controlled oven and a homemade separator. The function generator can generate pulsed DC with different wave shapes, such as square, sine, arbitrary. The voltage amplifier can amplify the input voltage to 2000 times higher. The maximum voltage and frequency created from this equipment are $10 \mathrm{kV}$ and $7.5 \mathrm{kHz}$. A temperature-controlled oven is used to achieve elevated temperature conditions. In this paper, the ranges of the operating temperature, voltage, and frequency are $25-90^{\circ} \mathrm{C}, 0-1 \mathrm{kV}$ and $10-5,000 \mathrm{~Hz}$.

\section{Water-in-oil emulsions}

The water-in-sunflower oil emulsions are made with $0.1 \%$ (wt.) Sodium Dodecyl Sulfate (SDS) surfactant and either $5 \%$ or $10 \%$ water.
The mixtures are mixed by an ultrasonic processor with $250 \mathrm{~W}$ power for 12 minutes to make water-in-sunflower oil emulsions.

\section{Design of electrodes and separation tube}

There are several factors affecting electro-coalescence, such as a type of current flow, voltage, frequency, electrode configuration, etc. Different types of current flow have been used for the electrocoalescence method: AC, DC and pulsed DC [16-19]. DC is efficient in promoting droplet coalescence but usually causes electrolytic corrosion. Therefore, it is rarely used in water-oil separation nowadays. AC was the most common method used in crude oil emulsion due to its tolerance to high water concentrations before the pulsed DC was introduced. Pulsed DC was proposed with insulated electrodes for high water content emulsions and has been extensively used since 1980s [2,20]. Pulsed DC provides a marked performance advantage over AC or DC power [2,21]. While pulsed DC has different types of wave shapes (e.g. sine, square, ramp, pulse, noise waves), square wave shape shows the highest efficiency [22]. Therefore, the square wave of pulsed DC is used for all the tests in our experiment. 
a.

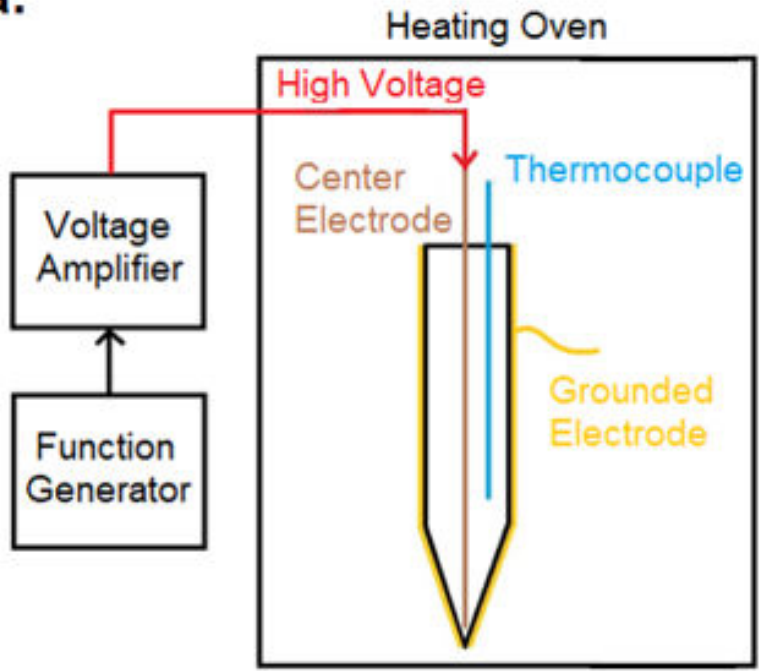

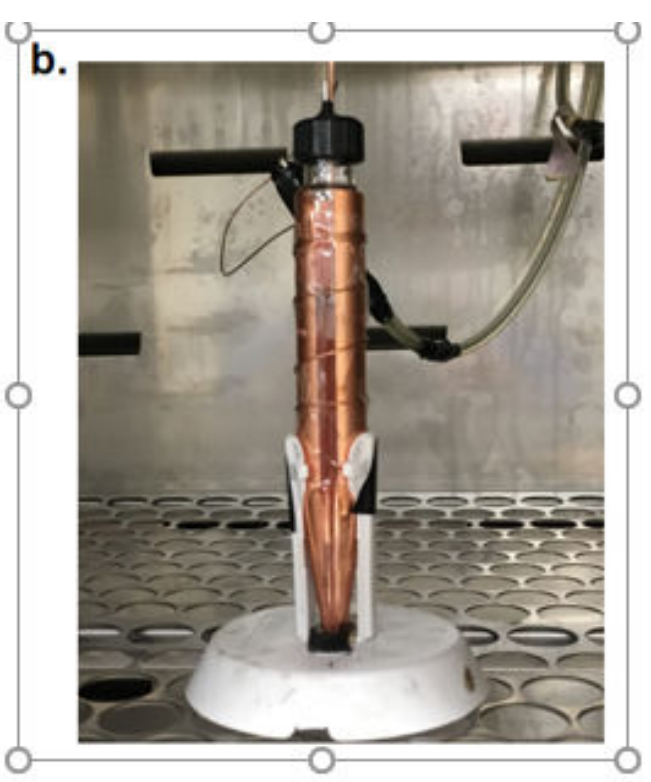

Figure 4: Experimental Setup for water-in-oil emulsion separation: (a) Overall diagram, (b) Cone-shaped cylindrical separation tube equipped with coaxial electrodes.

Coalescers are devices that are primarily used for liquid separation. For an electric coalescer, the design for electrodes is one of the key factors. There are two types of electrodes: uninsulated electrodes and insulated electrodes. An uninsulated electrode may cause short-circuit problem [23]. The chance of the electric short-circuits is significant when the water concentration is above $20 \%$. Since the crude oil may contain water concentration above $20 \%$, it may not be a wise choice to use the uninsulated electrode. On the other hand, insulated electrodes have electrical breakdown strength to withstand the voltage. This can reduce electrical potential at the electrode emulsion interface [24-29] and enhance separation efficiency [30]. Therefore, insulated electrodes are used for all the separation tests in our experiment.

The configuration (e.g. plate or cylinder) of electrodes can also affect separation performance due to their effects on the electric fields. As shown in Figures $5 \mathrm{a}$ and $5 \mathrm{~b}$, flat plate electrodes would create uniform electric fields, while cylindrical electrodes can generate nonuniform electric fields inside the separation tube. Non-uniform electric fields can enhance the water-oil separations compared to uniform fields [31-34]. In addition to generating non-uniform electric fields, cylindrical electrodes can create electric fields that cover most of the space in the separation tube. Therefore, in this paper, instead of using two flat copper plate electrodes, cone-shaped cylindrical electrodes are used in our experiment. The shape of a separator is another key factor. In this paper, a long and thin cylindrical tube is fabricated as the separator. By this design, the distance between the centre and surrounded electrodes can be minimized. This reduced gap brings a higher strength of electric fields and promotes the separation. The temperature of the emulsion can be measured by a thermocouple immersed in the emulsion.

A cylindrical conical glass tube, copper foils, and wires are used to design the cone-shaped separator. The dimensions of the conical separation tube are $128 \mathrm{~mm}$ height, $17 \mathrm{~mm}$ of the outer diameter and $13 \mathrm{~mm}$ of inner diameter. The volume of the separation tube is around
$15 \mathrm{~mL}$. A cap is used to cover and seal the separator. The separation tube is surrounded by the copper roll, which plays a role as a ground electrode. A central electrode and a thermocouple, which are insulated by glass capillary tubes, are fixed by going through the cap. The diameter of the center copper electrode is $1 \mathrm{~mm}$. A separation tube is supported by a cone-shaped tube holder.

\section{Measurement uncertainty}

The measurement uncertainty has significant influences on the assessment of the results. The measurement device, Karl Fischer Titrator, shows less than $0.5 \%$ of standard deviation with the range of $1-10 \%$ of water concentrations. Therefore, the uncertainties of measurement devices are negligible. However, a human experimental error may affect the results. Even though the tests are performed in same conditions, measurement of the separation speed and the water concentrations can show minor differences. Based on the results of repeated tests, the experimental test error can be less than $\pm 10 \%$.

\section{Experimental Results}

\section{Comparisons of heating, electrostatic, and combined methods}

Water-in-oil emulsions may be separated by either heating or electrostatic method alone. Three different tests with $10 \%$ water-insunflower oil emulsion are executed to compare the heat treatment, electrostatic method, and the combined method of these two. The experiments are carried out in the homemade separator using an experimental setup as shown in Figure 5. The water-oil separations observed with these three conditions are indicated in Figure 6a, and the residual water concentrations (\%) in the separated oil phase after 10-minute tests are listed in Table 1 . In the figures, red arrows indicate the interfaces between the separated water and oil phases. For both 
heating and electrostatic tests, the residual water concentrations (\%) in the separated oil phase are around 5\%. This indicates that only about $50 \%$ of the water is separated out of the emulsion by the heating or electrostatic method alone. Moreover, the water phase is not fully transparent when separated by only heat treatment as can be seen in the figure. However, when the combined method is used, the amount of separated water increases significantly. Around $90 \%$ of the water is separated as shown in Table 1 . Therefore, the combination of heating and electrostatic methods can bring a significant improvement in the quality of water separation.

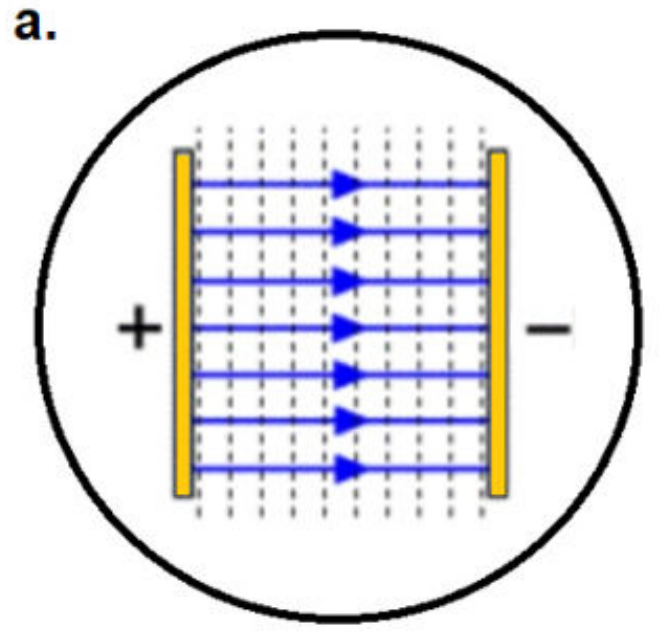

Figure 5: Different electric fields generated by flat plates and cylindrical electrodes: (a) Flat plate electrodes generate uniform electric fields, (b) Cylindrical electrodes generate non-uniform electric fields.

\section{Temperature dependence}

The speed and quality of the water-oil separation by the combined method may be affected by several factors: temperature, voltage, and frequency. Temperature dependence of the water-in-oil emulsion separation is investigated by executing separation tests at seven different temperatures: $25^{\circ} \mathrm{C}, 40^{\circ} \mathrm{C}, 50^{\circ} \mathrm{C}, 60^{\circ} \mathrm{C}, 70^{\circ} \mathrm{C}, 80^{\circ} \mathrm{C}$ and $90^{\circ} \mathrm{C}$. Other conditions remain constant during the tests. The applied voltage and frequency are $1 \mathrm{kV}$ and $50 \mathrm{~Hz}$ with a square wave. Figure $6 \mathrm{~b}$ indicates the separations of the emulsion during the test at four different operating temperatures: $25^{\circ} \mathrm{C}, 50^{\circ} \mathrm{C}, 70^{\circ} \mathrm{C}$ and $90^{\circ} \mathrm{C}$. At room temperature, $25^{\circ} \mathrm{C}$, no obvious separation is observed until 5 minutes after applying voltage. However, at $90^{\circ} \mathrm{C}$, the water-oil separation is observed in less than 1 minute. The amount of separated water increases during the test. Based on the test results, the water/oil separation time is reduced by $5-10$ times at $90^{\circ} \mathrm{C}$ compared to the tests under the same electric field at the room temperature $\left(25^{\circ} \mathrm{C}\right)$. This reduced separation time by elevating temperature shows an agreement with the result of the theoretical calculations.

The water concentrations of the separated oil phase are measured after 10-minute tests. The results of residual water concentration in the separated oils, with the initial water concentration of $5 \%$ and $10 \%$, are indicated in Figure $7 \mathrm{a}$. At $25^{\circ} \mathrm{C}, 40-50 \%$ of the water is separated out from the emulsion for both $5 \%$ and $10 \%$ emulsions. The amount of separated water also increases as the operating temperature increases. Over $90 \%$ of the water is separated at the highest temperature (i.e., $90^{\circ} \mathrm{C}$ ) with the residual water concentration around $0.8-0.9 \%$ in the separated oil phase. Therefore, the quality of the separated oil becomes higher with the increase of temperature. Especially, this high- b.

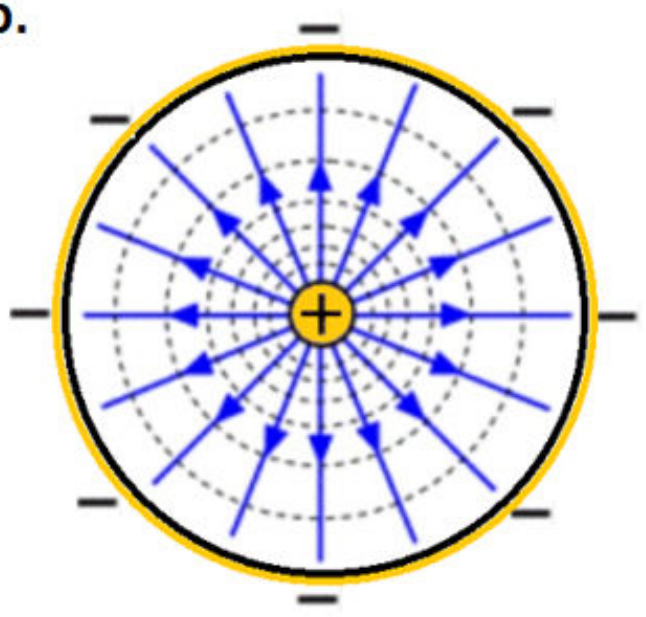

temperature treatment coupled with the electrostatic method can efficiently separate the emulsified oil (e.g. crude oil).
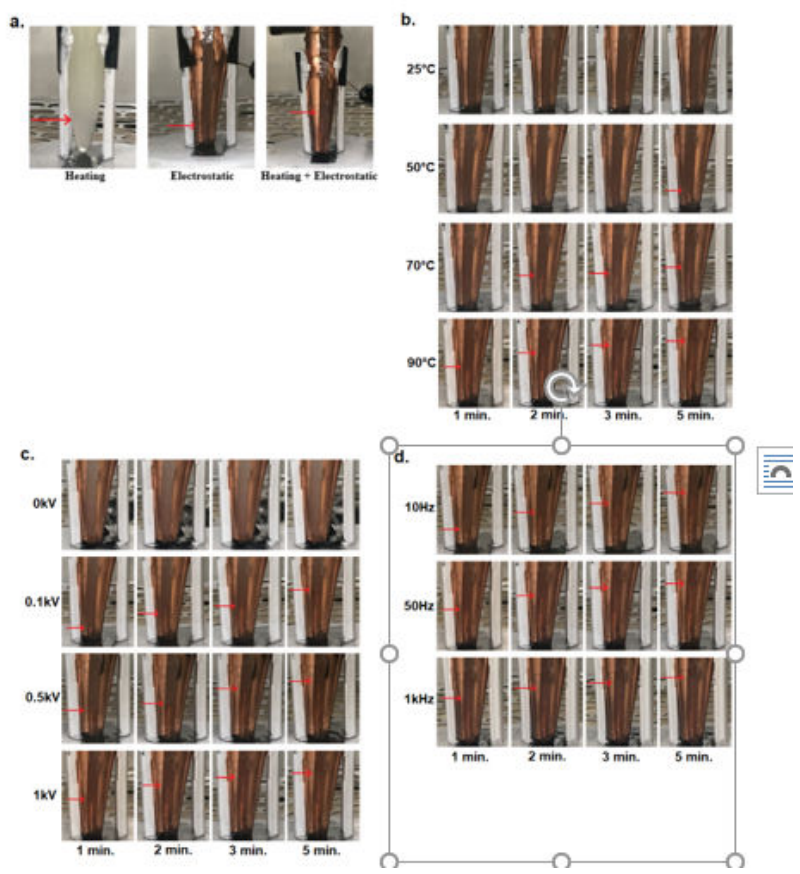

Figure 6: 10\% Separation of water-in-sunflower oil emulsion under different conditions: (a) Comparison of heating, electrostatic, and combined (heating + electrostatic) methods, (b) Separations by different temperatures, (c) Separations by different voltages, (d) Separations by different frequencies. 
Page 6 of 8

\begin{tabular}{|l|l|l|}
\hline \multirow{2}{*}{$10 \%$ Water-in-sunflower oil separations } & Residual water concentrations $(\%)$ in the oil phase \\
\cline { 2 - 3 } & $\mathbf{1 0}$ minutes & $\mathbf{6 0}$ minutes \\
\hline Heating Only & 4.49 & 2.75 \\
\hline$\left(90^{\circ} \mathrm{C}\right)$ & & \multirow{2}{*}{0.89} \\
\hline Electrostatic Only & 5.36 & \\
\hline$\left(25^{\circ} \mathrm{C}, 1 \mathrm{kV}, 1 \mathrm{kHz}\right)$ & & 0.48 \\
\hline Heating + Electrostatic & 0.94 & \\
\hline$\left(90^{\circ} \mathrm{C}, 1 \mathrm{kV}, 1 \mathrm{kHz}\right)$ & & \\
\hline
\end{tabular}

Table 1: Water concentrations of separated oil phase with the extended separation time.

\section{Voltage dependence}

The dependence of voltage is also investigated for the electrostatic separation. Five different voltages $(0 \mathrm{kV}, 0.1 \mathrm{kV}, 0.3 \mathrm{kV}, 0.5 \mathrm{kV}, 1 \mathrm{kV})$ are applied to the water-in-sunflower oil emulsions while all other conditions remain the same. The operating temperature is $90^{\circ} \mathrm{C}$, and the frequency of the electric field is $50 \mathrm{~Hz}$. The observed water-oil separations are shown in Figure 6c. No separated water phase is observed for the test with zero voltage (i.e., heating only) in five minutes. In contrast, the separations of water phase are observed when a voltage is applied to the system. As the applied voltage is increased from $0.1 \mathrm{kV}$ to $1 \mathrm{kV}$, the separation of water is also expedited. Figure $7 \mathrm{~b}$ presents the residual water concentrations in the separated oil phase after the 10-minute test. As shown in Figure 7b, the increase of voltage can deliver a higher quality of the separated oil phase with residual water concentration less than $1 \%$. For example, the application of $1 \mathrm{kV}$ voltage reduces the separation time by 5-10 times and decreases the residual water concentration by 4-5 times when compared to the test without applying voltage. a.

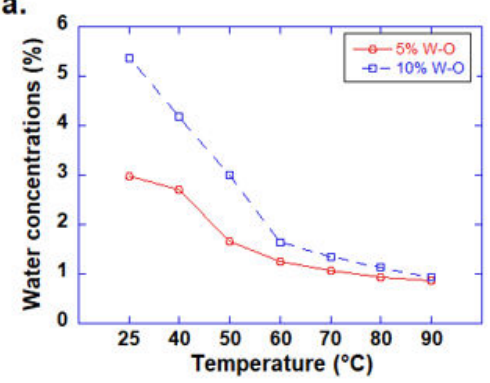

b.

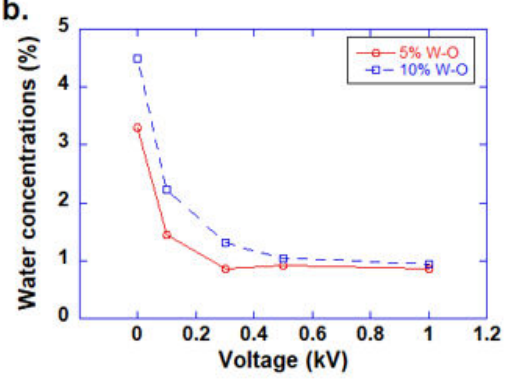

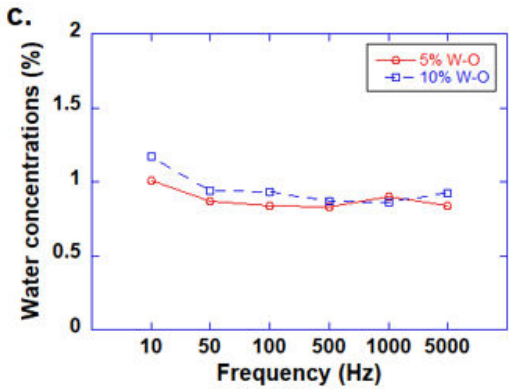

Figure 7: Water concentrations of separated oil phase for different conditions: (a) Temperature dependence, (b) Voltage dependence, (c) Frequency dependence.

\section{Frequency dependence}

The separation tests to determine the frequency dependence are executed with six different frequencies: $10 \mathrm{~Hz}, 50 \mathrm{~Hz}, 100 \mathrm{~Hz}, 500 \mathrm{~Hz}$, $1000 \mathrm{~Hz}, 5000 \mathrm{~Hz}$. Applied voltage and temperature are constant at 1 $\mathrm{kV}$ and $90^{\circ} \mathrm{C}$. The effect of frequency on the separation speed of waterin-oil emulsion is displayed in Figure 6d. As can be seen in the figure, the increasing of frequency may expedite the separation speed. However, the effect of the frequency on the separation speed is relatively small compared to the influences of temperature and voltage. Figure $7 \mathrm{c}$ indicates the residual water concentrations in the separated oil phase. As can be seen in the figure, the effect of increasing frequency becomes negligible when the frequency reaches a certain point (i.e., $50 \mathrm{~Hz}$ ). Water concentrations are also measured to be less than $1 \%$ when the applied frequency is over $50 \mathrm{~Hz}$.

\section{Testing time}

The extension of separation time may bring a higher quality to the separated oil. To illustrate that, separation tests of $10 \%$ water-insunflower oil emulsions for the extended time (1 hour) are conducted with heat treatment, an electrostatic method, and the combined method. The residual water concentrations (\%) in the separated oil after 1-hour tests are shown in Table 1. As can be seen in the table, the extension of separation time can reduce the water concentrations in separated oil for all the three methods. The electrostatic method brings much less water concentration $(0.89 \%)$ compared to the heating only method $(2.75 \%)$. However, the water concentration $(0.89 \%)$ of this one-hour electrostatic test is almost the same as the result of the combined method (0.94\%) with 10 minutes. Therefore, the combined method can reduce the separation time substantially with similar oil quality. This reduced separation time can also deliver a less energy consumption. Finally, when the testing time is increased to 1 hour for the combined methods, the water concentration was decreased around 
$0.5 \%$. Therefore, the combined method can achieve not only a higher quality of separated oil but also a reduction of the separation time.

\section{Energy consumption}

As shown in the previous results, the increase in voltage and frequency can enhance the separation quality and reduce the separation time. However, when the voltage or frequency reaches a certain point, the effect of increasing becomes negligible. The power consumptions with different voltage and frequency are indicated in Figures $8 \mathrm{a}$ and $8 \mathrm{~b}$. Therefore, it is also important to find the optimal values of voltage and frequency to minimize the unnecessary use of power consumption. In this system, the optimum voltage and frequency may be chosen to be $0.5 \mathrm{kV}$ and $50 \mathrm{~Hz}$. In addition, $10 \%$ water-in-sunflower oil emulsion shows a little higher energy consumption than 5\% emulsion as shown in Figures $8 \mathrm{a}$ and $8 \mathrm{~b}$ because the resistance of the emulsion reduces with the increase of water concentration. The optimum voltage and frequency can be changed for different separation systems because it is also affected by the configuration and dimensions. a.

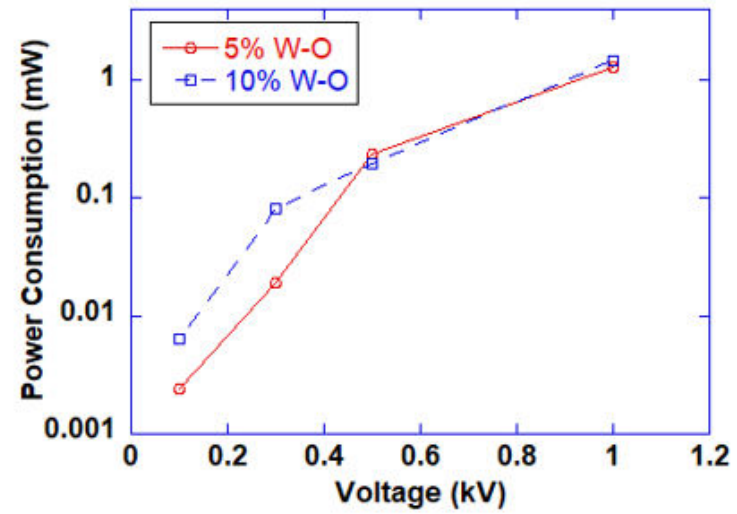

b.

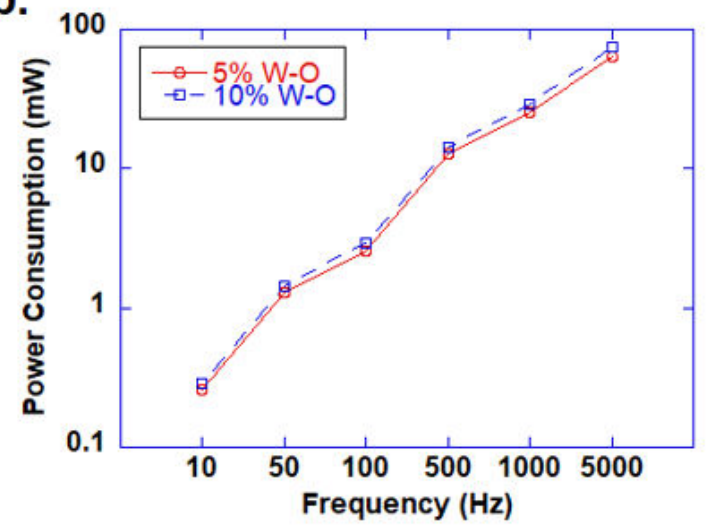

Figure 8: Power consumptions of the system under different conditions: (a) Voltage dependence, (b) Frequency dependence.

\section{Discussion}

The combination of heating and electrostatic methods is more effective than either method alone for the water-oil separation. The separation of the water-in-oil emulsion in electric fields is accelerated at the elevated temperature because the elevated temperature reduces the viscosity of emulsion, which makes the coalescing more rapid. The influences of elevating temperature for water/oil separation by electrostatic force are also theoretically investigated. Theoretical calculations show that the precipitation and collision times of water droplets are decreased by elevating temperature or electric strength. For instance, the water/oil separation speed is reduced by 5-10 times at high temperature $\left(90^{\circ} \mathrm{C}\right)$ when compared to the same process at room temperature $\left(25^{\circ} \mathrm{C}\right)$. These results are experimentally validated by executing water/oil separation tests with different temperatures and voltages. For example, increasing the operating temperature to $90^{\circ} \mathrm{C}$ can reduce the separation time by $5-10$ times and decrease the residual water concentration by 5-6 times when compared to the results under the same electric field at $25^{\circ} \mathrm{C}$. In addition, the application of the $1 \mathrm{kV}$ voltages can decrease the separation time by 5-10 times and reduce the residual water concentration by 4-5 times when compared to the test without electric field but with the same heating condition.

\section{Acknowledgment}

The authors acknowledge the support of NSF (CBET1336778) and ARPA-E (DEAR0000585).

\section{Conclusion}

In this separation study, the separated oil phase contains normally less than $1 \%$ of water after only a 10 -minute separation using the combined methods (i.e., heat treatment + electrostatic method). Temperature and voltage are significant factors that influence the speed and quality of separation, while frequency shows less effect. By increasing the temperature, voltage, and frequency, we may improve the quality of separated oil with reduced separation time.

Once the voltage and frequency reach certain points, the improvement of water-in-oil emulsion separation becomes negligible. Therefore, establishing the optimum voltage and frequency are important to balance the energy consumptions and separation efficiency. By estimating the power consumption, the optimum condition of our system is found to be $0.5 \mathrm{kV}$ and $50 \mathrm{~Hz}$ with $90^{\circ} \mathrm{C}$ of heating. In this condition, the water-oil separation is observed in around 30 seconds after applying the voltage and the amount of separated water keep increasing during the experiment. After a 10minute test, the water concentration of the separated oil is about $0.8 \%$. The result can be further increased to be around $0.5 \%$ with an extended separation time of 1 hour.

\section{References}

1. Boxall JA, Koh CA (2010) Measurement and calibration of droplet size distributions in water-in-oil emulsions by particle video microscope and a focused beam reflectance method. Ind Eng Chem Res 49: 1412-1418.

2. Eow J (2002) Electrostatic enhancement of coalescence of water droplets in oil: A review of the technology. Chem Eng J 85: 357-368. 
Citation: Lee HS, Zheng C, Yang B (2018) Separation of Water-in-Oil Emulsions by Electrostatic Field at the Elevated Temperature. J Appl Mech

Page 8 of 8

3. Zhang L, Zhong Y, Cha D, Wang P (2013) A self-cleaning underwater super-oleophobic mesh for oil-water separation. Sci Rep 3: 1.

4. Wang CF, Huang HC, Chen LT (2015) Protonated melamine sponge for effective oil/water separation. Sci Rep 5: 1.

5. Moroi Y (1988) Relationship between solubility and micellization of surfactants: The temperature range of micellization. Progr Colloid \& Polymer Sci 77: 55-61.

6. Eow JS, Ghadiri M (2003) Drop drop coalescence in an electric field: the effects of applied electric field and electrode geometry. Colloids and Surfaces A: Physicochem Eng Aspects 219: 253-279.

7. Sjoblom J, Urdahl O (1992) Stabilization and destabilization of water-incrude oil emulsions from the Norwegian continental shelf. Adv Colloid Interface Sci 41: 241-271.

8. Spiecker PM, Kilpatrick PK (2004) Interfacial rheology of petroleum asphaltenes at the oil-water interface. Langmuir 20: 4022- 4032.

9. Hosseini M, Shahavi MH (2012) Electrostatic enhancement of coalescence of oil droplets (in nanometer scale) in water emulsion. Chin J Chem Eng 20: 654-658.

10. Bresciani AE (2010) Coalescence of water droplets in crude oil emulsions: Analytical solution. Chem Eng Technol 33: 237-243.

11. Giljarhus N (2011) Numerical investigation of electrostatically enhanced coalescence of two drops in a flow field. ICDL.

12. Neelamegam P, Krishnaraj S (2011) Estimation of liquid viscosities of oils using associative neural networks. Indian J Chem Tech 18: 463-468.

13. Esteban B, Riba J, Baquero G, Rius A, Puig R (2012) Temperature dependence of density and viscosity of vegetable oils. Biomass and Bioenergy 42: 164-171.

14. Semancik P (2007) Dielectric analysis of natural oils. Acta Electrotechnica et Informatica p: 7.

15. Wu P, Qiao R (2011) Physical origins of apparently enhanced viscosity of interfacial fluids in electrokinetic transport. Phys of Flu 23: 072005.

16. Waterman LC (1965) Electrical coalescers. Chem Eng Progr 61: 51-57.

17. Galvin CP (1984) Design principles for electrical coalescers. Extraction 88 101-113.

18. Cottrell FG, Speed JB (1911) Separating and collecting particles of one liquid suspended in another liquid. US Patent 987114.
19. Cottrell FG, Speed JB (1911) Separating and collecting particles of one liquid suspended in another liquid. US Patent 987115.

20. Taylor, S.E. (1996) Theory and practice of electrically-enhanced phase separation of water-in-oil emulsion. Chem Eng Res Des 74: 526-540.

21. Pelleymounter D, Carter D (2012) The pulsed-DC advantage: Improve film quality and reduce downtime in reactive sputtering applications. 55th Annual Technical Conference Proceedings, Santa Clara, USA.

22. Lesaint C, Glomm WR (2009) Dehydration efficiency of AC electrical fields on water-in-model-oil emulsions. Colloids Surf. A: Physicochem Eng Aspects 352: 63-69.

23. Feng G, Huang J, Sumpter B, Meunier V, Qiao R (2010) Structure and dynamics of electrical double layers in organic electrolytes. Phys Chem Chem Phys 12: 5468.

24. Bailes PJ, Larkai SK (1986) Electrostatic separation of liquid dispersions. UK Patent No. 2171031A.

25. Winslow JD (1977) Electrical treater with A.C.-D.C. electrical field. US Patent No. 4049535

26. Wolfe H (1944) Method and apparatus for electrically treating fluids. US Patent No. 2364118.

27. Hsu EC, Li NN (1983) Electrodes for electrical coalescence of liquid emulsions. US Patent No. 4415426.

28. Bailes PJ (1996) Resolution of emulsions. US Patent 5: 580-464.

29. Qiao R (2006) Control of electroosmotic flow by polymer coating: Effects of the electrical double layer. Langmuir 22: 7096-7100.

30. Fjeldly TA, Hansen EB, Nilsen PJ (2008) Novel coalescer technology in first-stage separator enables single-stage separation and heavy-oil separation. SPE Projects Facil Constr 3: 1-5.

31. Eow JS, Ghadiri M (2002) Electrostatic enhancement of coalescence of water droplets in oil: a review of the technology. Chem Eng J 85: 357-368.

32. Mandal B, Sirkar A (2012) Effects of geometry of electrodes and pulsating dc input on water splitting for production of hydrogen. IJRER 2.

33. AI-Besharah, JM, Salman OA (1987) Viscosity of crude oil blends. Ind Eng Chem Res 26: 2445-2449.

34. Luo S, Schiffbauer J, Luo T (2016) Effect of electric field non-uniformity on droplets coalescence. Phys Chem Chem Phys 18: 29786-29796. 